Original Paper http://ajol.info/index.php/ijbcs http://indexmedicus.afro.who.int

\title{
Atouts et contraintes de la pisciculture traditionnelle de Bandial (Casamance, Sénégal)
}

\author{
Ngor NDOUR ${ }^{1 *}$, Bienvenu SAMBOU ${ }^{2}$, Hamet DIADHIOU ${ }^{3}$ Hyacinthe SAMBOU $^{2}$ et \\ Maurice DASYLVA ${ }^{1}$
}

${ }^{1}$ Université Assane SECK de Ziguinchor, Département d'Agroforesterie, Sénégal.

${ }^{2}$ Université Cheikh Anta Diop, Dakar, Sénégal.

${ }^{3}$ Centre de Recherche Océanographique Dakar Thiaroye, Sénégal.

*Auteur correspondant ; E-mail :ngorndour@univ-zig.sn; Tél: + 2215533203

\section{REMERCIEMENTS}

Le collectif des auteurs remercient l'Université Assane Seck de Ziguinchor pour avoir financé l'étude diachronique de la pisciculture traditionnelle dans la zone d'étude.

\section{RESUME}

Socialement importante, la pisciculture traditionnelle est en déclin en Casamance. Contre cette décadence, cette étude promeut la connaissance et la relance de la pisciculture ancienne. Une analyse diachronique, des poissons élevés, est réalisée à l'aide d'enquêtes. Les données biométriques des poissons ont été collectées et les variables physicochimiques du milieu mesurées. La baisse de la diversité des poissons montre que deux espèces (Galeoïdes decadactylus et Arius heudeuloti) ne sont plus élevées à Bandial. La biomasse obtenue a variée entre 0,16 et 0,24 tonne de poissons/ha/étang. La régression allométrique $\left(\mathrm{Pt}=\mathrm{aLt}^{\mathrm{b}}\right)$ indique des pentes de croissance b, parfois majorantes chez Sarotherodon melanotheron et Hemichromis fasciatus. La croissance isométrique a été notée chez Sarotherodon melanotheron, Elops lacerta et les mulets. La croissance négative est notée chez Sarotherodon melanotheron à Yambathine, étang le moins favorable du terroir. L'embonpoint $(\mathrm{K})$ des espèces est compris entre 0,33 et $5,4 \mathrm{~g} / \mathrm{cm}^{3} . \mathrm{K} \geq 1,60 \mathrm{~g} / \mathrm{cm}^{3}$ indique d'excellentes conditions environnementales pour les carpes et les mulets. Les variables physico-chimiques du milieu et le potentiel biologique sont favorables à la relance de la pisciculture à Bandial. Il ressort que les conditions environnementales se prêtent au développement de la pisciculture traditionnelle en BasseCasamance.

(C) 2017 International Formulae Group. All rights reserved.

Mots clés : Etangs, poissons, diversité, conditions, croissance, biomasse.

\section{Assets and constraints of traditional fish breeding in Bandial (Casamance, Senegal)}

\begin{abstract}
Traditional fish breeding is in decline in Casamance despite its social importance. To remedy this decline, this study aimed to promote the knowledge and the revival of the traditional fish breeding. A diachronic analysis of breeded fish was carried out using surveys. The biometric data of the fish were collected
\end{abstract}


and the physicochemical variables of the environment measured. The decline in fish diversity showed that two species (Galeoïdes decadactylus and Arius heudeuloti) were no longer breeded in Bandial. The recorded biomass varied between 0.16 and 0.24 tons of fishes / ha / pond. The allometric regression $\left(\mathrm{Pt}=\mathrm{aLt}^{\mathrm{b}}\right)$ indicated greater slopes of growth for Sarotherodon melanotheron and Hemichromis fasciatus. An isometric growth was observed for Sarotherodon melanotheron, Elops lacerta and the mullets. A negative growth was noticed for Sarotherodon melanotheron at Yambathine in the least favorable pond in this area. The coefficient of condition $(\mathrm{K})$ of the species was comprised between 0.33 and $5.4 \mathrm{~g} / \mathrm{cm}^{3}$. In addition, $\mathrm{K} \geq 1.60 \mathrm{~g} / \mathrm{cm}^{3}$ indicated excellent environmental conditions for carps and the mullets. The physicochemical variables of the environment and the biological potential were favorable to the revival of traditional fish breeding in Bandial. From this study, we can conclude that environmental conditions are favorable for the development of fish breeding in LowCasamance.

(C) 2017 International Formulae Group. All rights reserved.

Keywords: ponds, fish, diversity, conditions, growth, biomass.

\section{INTRODUCTION}

Les mangroves sont aménagées pour développer la pisciculture et l'ostréiculture (Hem et al., 2001; Sow, 2005). En Asie, les règles et modalités de gestion des mangroves ont permis d'intégrer les palétuviers dans les systèmes de production aquacole. Cette gestion consiste à intégrer les palétuviers dans leur système de production aquacole. Dans un contexte de marché libéral, la plupart des systèmes actuels de production sont intensifiés et de type monoculture (Hussenot, 2004). Dans cette intensification, les systèmes de production aquacole générèrent des déchets azotés et phosphoriques; polluant l'environnement aquatique. Face à cette situation, les chercheurs proposent des procédés d'épuration des eaux usées issues de ce type l'aquaculture (Bergheim et Brinker, 2003; Hussenot, 2003; Piedrahita, 2003, Mikolasek et al., 2009b). Pourtant, un système de production intégrée aurait pu transformer de lui-même les déchets en ressources non nuisibles pour l'écosystème (Hussenot, 2003; FAO, 2006 ; Mialhe, 2010 ; Ewoukem, 2011 ; Laamiri, 2014). Dans un système aquacole intégré, plusieurs organismes se mettent ensemble pour la création d'un écosystème où l'interaction des différentes composantes diminue l'impact des déchets produits par le système sur l'environnement. Cette interaction permet aux organismes d'optimiser l'utilisation de l'espace, des nutriments et d'améliorer les rendements aquacoles (Neori et al., 2004; Mialhe, 2010).

En Afrique, la pêche «Acadja» pratiquée au Bénin, au Nigéria est une variante de ce système traditionnel intégré. Ces aménagements permettaient de créer de nouvelles niches écologiques pour les poisons. Au Sénégal, la consommation de poissons par habitant $(37 \mathrm{~kg} / \mathrm{an})$ est la plus élevée en Afrique (FAO, 2010). Cependant, bien que séculaire, la pisciculture n'est plus bien connue par les jeunes producteurs particulièrement en basse Casamance. Il s'y ajoute qu'ils rencontrent de multiples problèmes notamment le piégeage des poissons qui n'est pas un mode d'empoisonnement susceptible d'atteindre la charge critique d'un étang. Par ailleurs, ce mode d'encensement donne une faible production surtout lorsque la moisson du riz définit la date de récolte du poisson (Idée Casamance, 2007). Il existe donc une symbiose entre la riziculture et la pisciculture 
en Casamance (Cormier-Salem, 1990; Idée Casamance, 2007 ; Miller, 2010). Cette relation réduit la période de croissance des poissons dans les étangs (Idée Casamance, 2007). Par ailleurs, les producteurs ignorent les causes des changements environnementaux (acidité, salinité...) notés dans les étangs. Cette situation a favorisé le déclin de la pisciculture en basse Casamance (Miller, 2010). De surcroît, les revenus tirés de l'activité sont maigres et ne peuvent constituer une source de motivation des producteurs comme c'est le cas ailleurs selon Peltier et al. (2005). La main d'œuvre familiale nécessaire à l'entretien des étangs, n'est plus disponible alors que les systèmes hydro-agricoles ancestraux se détériorent de plus en plus dans la région (Idée Casamance, 2007). Cependant, certains pisciculteurs ont maintenu ce système de production sans que l'on ait une idée de la diversité des poissons élevés ni des conditions environnementales qui prévalent dans les étangs. Cette étude prend en charge ces questions afin d'examiner ce système de production pour la relance de la pisciculture traditionnelle en Basse Casamance.

\section{MATERIEL ET METHODES}

\section{Choix des étangs d'élevage}

Les étangs étudiés ont été choisis sur la base d'un recensement des unités de production fonctionnelle. Le recensement a été fait auprès des producteurs et le choix des étangs a tenu compte de leur utilisation et de leur accessibilité à l'échelle du terroir villageois de Bandial. Cinq étangs aménagés ont été recensés dont trois en cours d'utilisation au moment de l'enquête. Ces trois étangs ont été retenus pour faire l'état de la diversité des poissons élevés et des conditions environnementales des unités de production.

\section{Description des étangs piscicoles}

Le milieu d'étude est constitué des étangs Wawdjugué, Amind'éwole et Yambathine où la faune piscicole a été étudiée du mois juillet 2014 au mois de mars 2016. L'étang Wawdjugué est traversé par deux chenaux communiquant sur toute sa longueur. Le cours de chaque chenal est interrompu par une digue de hauteur moyenne $1,75 \mathrm{~m}$ et d'épaisseur moyenne 1,50 m. En période de reproduction des poissons, ces chenaux permettent aux géniteurs de remonter vers l'étang pendant la marée haute. Les pisciculteurs empoissonnent naturellement leur étang pendant cette période en entaillant les digues à divers endroits sur une profondeur moyenne de $62,5 \mathrm{~cm}$. Ces entailles créent une dénivellation entre les eaux du cours d'eau et le fond de chaque étang. L'étang Amind'éwole est aussi traversé par trois chenaux dont le plus large fait $8,5 \mathrm{~m}$. Ces derniers permettent aux poissons de rejoindre l'étang à partir des entailles effectuées à divers endroits où les géniteurs, à la recherche de sites de ponte, se regroupent en marée haute pendant la période d'empoisonnement. Yambathine est aussi traversé par deux chenaux qui favorisent la remontée des géniteurs vers l'unité de production. La section des digues permet aux producteurs de remplir les étangs d'eau salée et de les empoisonner à l'échelle de deux semaines. Amind'éwole, Wawdjugué et Yambathine font respectivement 26,92, 14,06 et 4,34 ha et ont une profondeur moyenne respective de $34,14 \mathrm{~cm}, 28,66 \mathrm{~cm}$ et $14,12 \mathrm{~cm}$. Les levées de surface réalisées à l'aide d'un GPS ont permis de localiser ces étangs (Figure 1). 


\section{Collecte des données}

La collecte des données sur la diversité des poisons dans les étangs auprès des producteurs de Bandial, a permis de lister les espèces élevées autrefois et celles élevées au cours de l'enquête. Cette enquête a aussi permis de recenser les étangs fonctionnels. Le choix des étangs à étudier a tenu compte de leur fréquence d'utilisation de leur accessibilité et du mode de gestion traditionnelle. Parmi les étangs, deux sont gérés par des GIE et Yabathine est géré par les membres d'une famille. Les espèces de poissons élevés dans ces étangs ont été recensées et identifiées à l'aide d'ouvrages de plusieurs auteurs (Diouf, 1991 ; Seret, 2011), en collaboration avec les pisciculteurs, lors de la récolte. Celle-ci se fait soit à l'aide d'une nasse, d'un épervier ou d'un filet maillant dérivant. Les paramètres de croissance des poissons et leur biomasse ont été mesurés à chaque pêche. Au plus 10 individus capturées ont fait l'objet d'une mesure biométrique (longueur totale et longueur standard en $\mathrm{cm}$ ) et de biomasse en gramme par catégorie de taille/ et par espèce (Figure 2).

A partir de la relation $\mathrm{Pt}=\mathrm{aLt}^{\mathrm{b}}$, le type de croissance des principales espèces de poisson a été déterminé en calculant la constante $a$ et la pente $b$ de la régression issue de la transformation de la relation: $\log _{10}(\mathrm{P})=$ $\log _{10}(a)+\operatorname{bLog}_{10}(\mathrm{LT})$. La valeur de $b$ indique le type de croissance pour chaque espèce de poisson. Dans l'optique d'apprécier l'embonpoint des poissons, le facteur de condition a été calculer à l'aide de la relation $k=\frac{P t}{(L S)^{3}} \times 100$ où Pt est égale au poids total du poisson et LS sa longueur standard. Celuici permet de comprendre les impacts de la variation des conditions environnementales sur les espèces et de mesurer leur stade de développement physiologique (Oni et al., 1983). Pour mieux apprécier l'effet des conditions environnementales, le $\mathrm{pH}$, la salinité et la quantité d'oxygène contenu dans les eaux ont été mesurés dans les étangs à l'empoissonnement, à mi- élevage et à la récolte des poissons.

\section{Traitement des données}

Les données des étangs collectées au GPS ont été traitées à l'aide du logiciel Map Source. Elles ont permis de cartographier les étangs de la zone d'étude avec Arc GIS. La liste des espèces élevées dans chaque étang a été établie à la suite d'un recensement des espèces pendant les récoltes. La biomasse moyenne a été calculée à l'issue de la récolte et la biomasse correspondante mesurée. L'extrapolation des résultats a tenu compte des 4 jours de pêche à la nasse en moyenne par semaine. L'autre partie de la biomasse a été quantifiée au cours de la récolte massive qui marque la fin d'un cycle d'élevage. Une analyse en composantes principales (ACP) a permis de déterminer les corrélations entre les paramètres physicochimiques des étangs. Elle a aidé à mieux apprécier la distribution des poissons dans le plan factoriel F1\&F2 au regard de la contribution de ces variables dans la formation de axes de l'ACP. L'échelle d'interprétation fournit par IBGE (2005) a permis d'interpréter la variation des paramètres physico-chimiques du milieu. Par ailleurs, le facteur de condition (k) des espèces de poisson a permis de les classer en groupes ayant leur embonpoint quasiment identique. L'amplitude de la classe est déterminée par la méthode de Sturges (1926) et l'interprétation des résultats basée sur les travaux de Fournier (2012). Dans son approche, lorsque $\mathrm{K}$ est supérieur à 1,60 , les poissons sont dans d'excellentes conditions d'élevage, lorsque $\mathrm{k}=1,40$, les poissons sont dans de bonnes conditions d'élevage. Quand $\mathrm{k}=1,20$, les conditions d'élevage sont acceptables et quand $\mathrm{k}=1,00$, les conditions d'élevage sont considérées mauvaises. La pire des conditions d'élevage considérée très mauvaise correspond à $\mathrm{K} \leq 0,80$. 


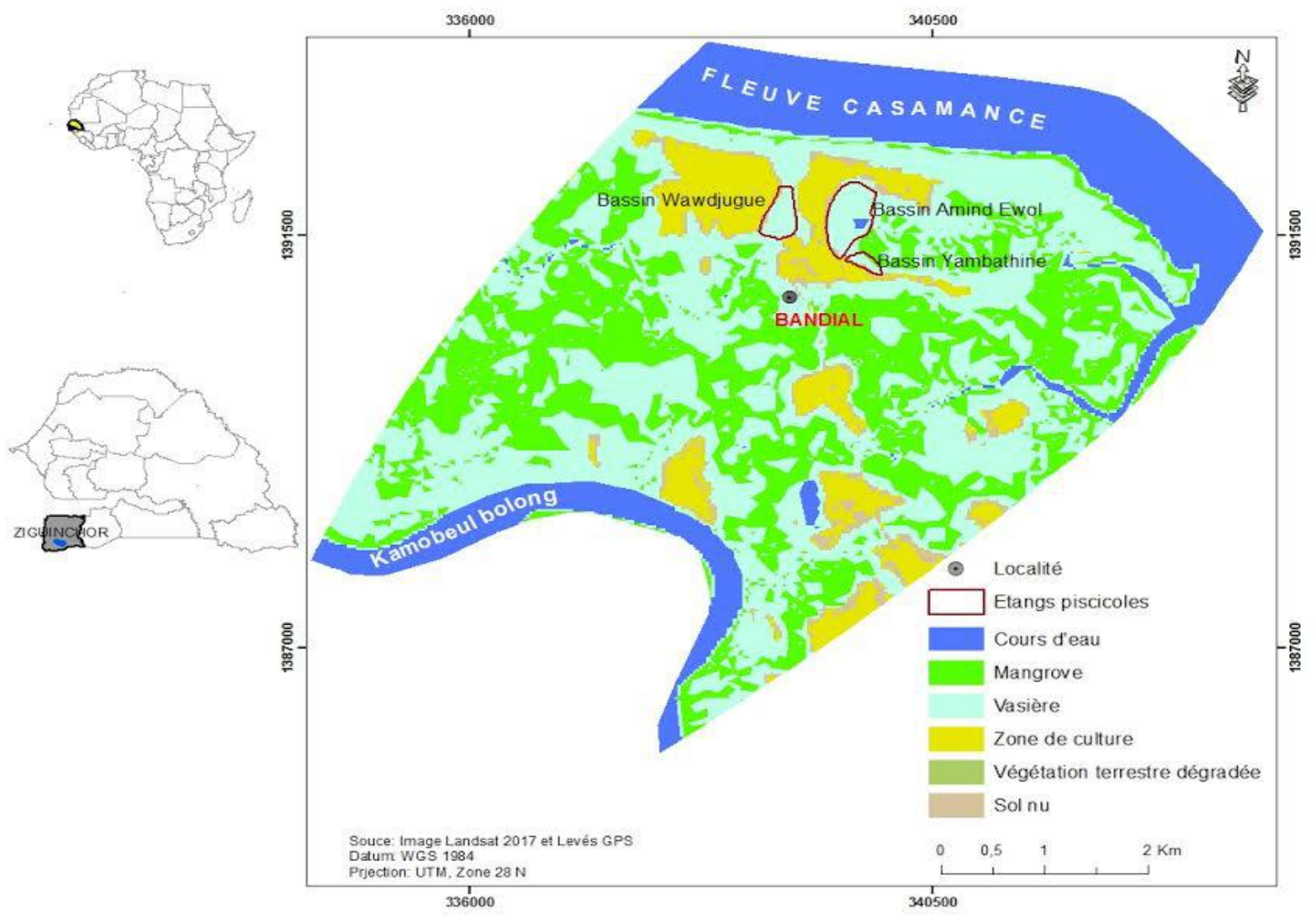

Figure 1 : Localisation des étangs piscicoles de Bandial.
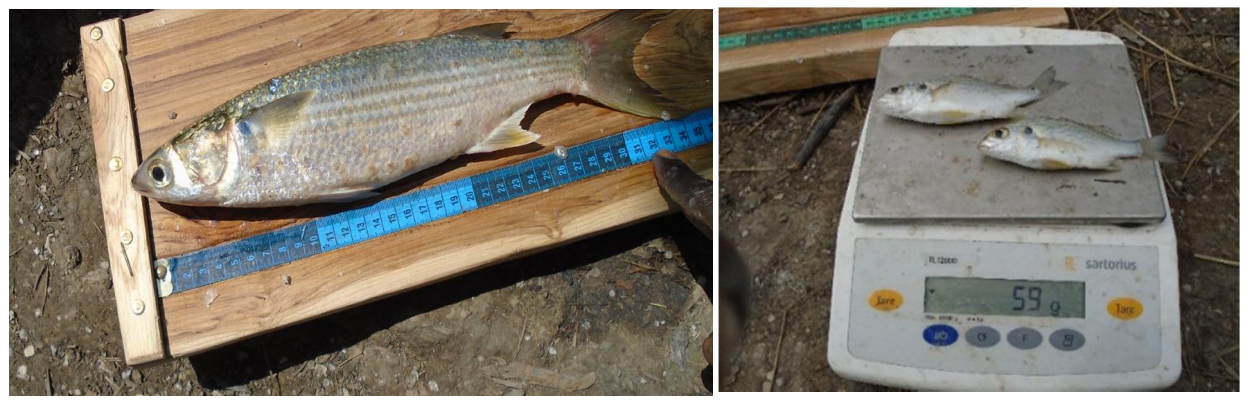

Figure 2: Mesure de la taille et de la biomasse des poissons. 
RESULTATS

Analyse diachronique de la diversité des poissons dans les étangs

L'analyse diachronique a permis de noter une régression de la diversité des poissons dans les étangs. Autrefois, 17 espèces de poissons étaient élevées à Bandial. Parmi ces poissons, 15 espèces le sont dans les trois étangs étudiés (Tableau 1). La seizième, Solea senegalensis n'a pas été signalée dans l'analyse diachronique et a été récolté en 2015-2016

Deux espèces de poissons dont le capitaine (Galeoides decadactylus) et le mâchoiron (Arius heudeleti) ne sont plus élevées dans les étangs. Les producteurs ont noté, dans le temps, une baisse de la diversité des espèces de poissons dans les étangs. Trente ans dans le passé, une diminution de la diversité des espèces de poissons a été notée dans les étangs alors que dans l'espace l'absence de ces deux espèces de poisson s'explique par l'abandon de la pisciculture dans les bassins autrefois aménagés à l'intérieur de la mangrove. Selon les producteurs, les espèces telles que Galeoides decadactylus et Arius heudeloti ne remontent pas au niveau des étangs relativement éloignés de la forêt de palétuviers. Autrefois, ces espèces remontaient les chenaux pour intégrer les bassins aménagés dans la forêt de mangrove à proximité du fleuve Casamance et du Kameubeul bolong.

\section{Diversité des espèces de poisson dans les étangs fonctionnels}

Lors de la récolte du poisson en 20142015 et en 2015-2016, la liste des poissons élevés a été établie pour chaque étang (Tableau 2). Il ressort que Wawdjugué et Amind'éwole sont plus diversifiés en poissons que Yambathine.

$82,25 \%$ des espèces élevées dans les étangs de Bandial sont rencontrées à Wawdjugué et 62,5\% à Amind 'éwole en 20142015. En 2015-2016, Amind'éwole comptait
$82,25 \%$ des espèces récoltées dans les étangs. Sphyraena barracuda considérée comme une espèce qui n'est plus élevée dans les étangs a été récoltée en 2015-2016. L'étang le moins diversifié en poissons est Yambathine avec $43,75 \%$ des espèces élevées entre 2014 et 2016. Pourtant, la distance moyenne qui sépare les étangs est de $49 \mathrm{~m}$ entre Amind'éwole et Yambathine et de $350 \mathrm{~m}$ entre Amind'éwole et Wawdjugué. Celle-ci n'explique pas fondamentalement la diversité des espèces observées entre les étangs. L'envergure des bolons et le nombre de chenaux connectés aux étangs pourraient être un des facteurs explicatifs de cette situation.

\section{La production piscicole par étang}

La biomasse de poissons élevés varie en fonction des années et des étangs. A Amind'éwole, il y' a eu une augmentation de la biomasse totale en 2015-2016 (Figure 3). C'est l'étang qui a enregistré la plus grande production de biomasse pendant la durée de l'étude. En termes de rendement, Amind'éwole a produit en moyenne 0,16 à 0,21 tonne de biomasse à l'hectare respectivement en 2014-2015 et 2015-2016. A Wawdjugué, le rendement $(0,22$ tonnes à l'hectare) de la biomasse était le plus important à l'échelle des trois étangs en 20142015. Cette même situation a été observée à Yambathine avec un rendement de 0,24 tonne de biomasse à l'hectare. Il ressort donc que les étangs les plus petits ont eu un meilleur rendement la première année de l'étude.

\section{Analyses morpho-métriques des poissons}

L'analyse morpho métrique des poissons à l'échelle de la pêche à la nasse et de la pêche massive indique qu'à Wawdjugué le groupe des mulets, Sarotherodon melanotheron et Elops lacerta sont les principales espèces récoltées. Lors de la pêche à la nasse les mulets récoltés sont distribués dans des classes de tailles: [6-10,87[cm $(23,16 \%)$, les classes $[10,87-15,65[\mathrm{~cm}$ et 
[15,65-20,43[cm qui comptent chacune $7,37 \%$. L'essentiel des mulets pêchés à la nasse est constitué de fretins et de juvéniles. $\mathrm{Au}$ cours de la pêche massive les mulets sont représentés dans les classes $|20,43-25,21| \mathrm{cm}$ $(41,05 \%),[25,21-29,99[\mathrm{~cm}(15,79 \%),[29,99$ $34,77[\mathrm{~cm}(3,16 \%$ et $[34,77-39,55[\mathrm{~cm}(2,11 \%)$. Les mulets de la pêche massive sont constitués d'individus de biomasse moyenne de $148,28 \mathrm{~g}$, de $239,33 \mathrm{~g}$, de $328 \mathrm{~g}$ et de $394 \mathrm{~g}$ dans l'ordre respectif des classes représentées. Les spécimens des quatre dernières classes représentent l'essentiel du poisson vendu $(62,52 \%)$ sur le marché de Ziguinchor à la fin de l'élevage. La deuxième espèce Sarothrerodon melanotheron est représentée dans les classes $[8-9,75[\mathrm{~cm}(6,67 \%)$, les classes: $[9,75-11,50[$ et $[11,50-13,25[\mathrm{~cm}$ avec chacune respectivement $13,33 \%$. Il ressort que la majorité des poissons pêchés est constituée de fretins de l'espèce puisque les biomasses moyennes des individus par classe sont respectivement de $13,5 \mathrm{~g}, 22 \mathrm{~g}$ et 40,5 g. Chez Elops lacerta, les classes représentées sont: [6-11,20[cm (2,86\%), [11,20-19,40[cm (20\%), $[19,40-24,50[\mathrm{~cm}(31,43 \%)$ lors de la pêche à la nasse. Les individus de biomasse plus importante sont récoltés lors de la pêche massive dans les classes de taille: [24,40$29,80[\mathrm{~cm}(17,14 \%) ;[29,80-35$ [cm $(25,71 \%)$ et $[35-40[\mathrm{~cm} \quad(2,86 \%)$. Les individus de biomasse plus élevée représentent $47,71 \%$ des prises de l'espèce et se vendent bien sur le marché de Ziguinchor. A Amind'éwole, le groupe des mulets, Sarotherodon melanotheron, Elops lacerta et Hemichromis fasiatus sont les principales espèces élevées. Les classes de taille représentées chez les mulets lors de la pêche à la nasse sont [7$12,18[\mathrm{~cm} \quad(27,94 \%), \quad[12,18-17,36[\mathrm{~cm}$ $(26,73 \%), \quad[17,36-22,54 \mathrm{~cm} \quad(9,81 \%)$, et $[22,54-27,72[\mathrm{~cm} \quad(8,30 \%)$. Les poissons pêchés sont aussi des juvéniles du groupe puisque les deux premières classes représentent $(54,67 \%)$ des captures. Pendant la pêche massive, les clases représentées sont $[27,72-32,9[\mathrm{~cm} \quad(16,91 \%), \quad[32,9-38,08[\mathrm{~cm}$ $(8,61 \%),[38,08-43,26$ [cm $(1,51 \%)$, et $[43,26-$ $48,44[\mathrm{~cm}(0,15 \%)$. Les gros mulets sont récoltés au cours de cette pêche avec des poids moyens respectifs par classe de 175,04 $\mathrm{g}$, de $256,44 \mathrm{~g}$ de $374,54 \mathrm{~g}$ et de $586,3 \mathrm{~g}$. Le pourcentage des gros mulets est certes faible par classe, mais la qualité du poisson récolté se vend aisément à Ziguinchor. Les producteurs estiment que l'essentiel de leurs revenus leur vient de la vente des gros sujets de Mugil cephalus et de Liza grandisquamis récoltés en fin d'élevage. Chez Sarotherodon melanotheron, les classes représentées sont [7-8,50[cm (16,81\%), [8,50-10,6[c m $(6,72 \%)$, $[10,6-12,7[\mathrm{~cm} \quad(6,72 \%)$ et $[12,7-14,6[\mathrm{~cm}$ $(8,40 \%)$. Il apparaît clairement que l'essentiel $(67,22 \%)$ des individus de l'espèce est récolté à la nasse. La dernière capture est constitué des juvéniles de l'espèce avec des biomasses moyennes respectives par classe de $10,15 \mathrm{~g}$, de $18,10 \mathrm{~g}$ et de $30,13 \mathrm{~g}$. Les premières prises regroupent majoritairement les fretins $(52,10 \%)$ et les juvéniles $(15,12 \%)$ de la biomasse récoltée. Les gros sujets représentent $32,77 \%$ des prises et contribuent à l'amélioration des revenus des producteurs. Chez Elops lacerta les individus sont représentés dans plusieurs classes dont celles: $[15-20,61[\mathrm{~cm} \quad(6,73 \%), \quad[20,61-26,22[\mathrm{~cm}$ $(14,42 \%)$ et la classe $[26,22-31,83[\mathrm{~cm}$ $(10,58 \%))$, Ces trois classes représente $31,73 \%$ de la pêche à la nasse. Les individus qui les constituent ont une biomasse moyenne respective par classe de 47,28 g, 96,87 g et de 150,82 g. Ils sont constitués des juvéniles et d'adultes de l'espèce. Les gros sujets sont récoltés lors de la pêche massive et sont représentés dans les classes suivantes: $[31,83-$ $37,44[\mathrm{~cm} \quad(17,31 \%), \quad[37,44-43,05[\mathrm{~cm}$ $(30,77 \%), \quad[43,05-48,66[\mathrm{~cm} \quad(15,38 \%) \quad$ et [48,66-54,27[|cm (4,81\%). L'analyse morpho métrique montre que l'essentiel des prises $(68,27 \%)$ de l'espèce a lieu lors de la pêche massive. Les gros individus de Elops lacerta sont pêchés lors de la récolte finale et 
comprennent des individus de taille $50 \mathrm{~cm}$. La biomasse moyenne obtenue par individus respectivement dans les différentes classes de taille est de $221,17 \mathrm{~g}, 408,78 \mathrm{~g} ; 625,56 \mathrm{~g}$ et 764,4 g. La taille atteinte par l'espèce à Amind'éwole semble intéressante puisque proche de la taille des gros individus pêchés en mer. D'ailleurs, le produit rapporte des revenus substantiels aux producteurs. Parmi les principales espèces notées à Amind 'éwole, Hemichromis fasciatus est représenté dans les classes: $[8,50-10,75[\mathrm{~cm} \quad(18,11 \%),[10,75-$ $13,08[\mathrm{~cm} \quad(35,43 \%), \quad[13,08-15,37[\mathrm{~cm}$ $(11,81 \%), \quad[15,37-17,66[\mathrm{~cm} \quad(14,96 \%)$. Ces individus, constitués de juvéniles et de sujets matures, représentent $(80,31 \%)$ des prises de l'espèce. Les plus gros individus sont récoltés lors de la pêche massive et sont représentés dans les classes : [17,16-19,95[cm $(10,24 \%)$, $[19,95-22,24[\mathrm{~cm}(8,66 \%)$ et $[22,24-24,53[\mathrm{~cm}$ $(0,79 \%)$. Les gros sujets, rares lors de la récolte finale, représentent $19,69 \%$ des prises de l'espèce. La pêche à la nasse semble plus adaptée à la capture des individus de l'espèce qui cherche à retourner dans le fleuve à la fin de la saison des pluies à partir des drains de connexion installés entre les étangs et les chenaux du Kamobeul bolong ou du fleuve Casamance

A Yambathine, Sarotherodon melanotheron est représentée dans les classes : $\quad[5,5-6,64[\mathrm{~cm} \quad(36,96 \%), \quad[6,64-$ $7,78[\mathrm{~cm}(0 \%), \quad[7,78-8,92[\mathrm{~cm} \quad(57,83 \%)$, [8,92-10,06[ cm (4,78\%), [10,06-11,2[ cm $(0 \%),[11,2-12,34[\mathrm{~cm}(0 \%),[12,34-13,46[\mathrm{~cm}$ $(0,46 \%)$. Chez l'espèce, la structure de la population est discontinue puisque trois classes ne sont pas représentées. Les prises sont essentiellement constituées de fretins (99,57\%). Dans cet étang, les mulets sont représentés dans les classes: $[6,5-8,89[\mathrm{~cm}$ $(70,58 \%),[8,89-11,28[\mathrm{~cm}(11,76 \%),[11,28-$ $13,67[\mathrm{~cm} \quad(0 \%), \quad[13,67-16,06[\mathrm{~cm} \quad(0 \%)$, $[16,06-18,45[\mathrm{~cm}(11,76 \%)$ et $[18,45-20,84[$ $\mathrm{cm}(6,47 \%)$ lors de la pêche à la nasse. Il ressort que la taille est une structure discontinue chez le groupe des mulets. Cette rupture témoigne de l'absence d'individus de grande taille dans l'étang à toutes les phases de l'élevage des poissons. Ainsi, l'essentiel des prises effectuées à la nasse est constitué de fretins et de juvéniles toutes espèces confondues. Les gros individus étant absents de Yambathine, la pêche massive n'y est d'ailleurs pas pratiquée par la famille propriétaire de l'étang. Le poisson récolté est vendu à l'échelle du village de Bandial et des villages environnants.

\section{Analyse de la relation poids et taille des poissons \\ La relation entre le poids et la taille des} poissons a été étudiée par étang. Cette relation a été analysée chez les mulets et chez Sarotherodon melanotheron, Elops lacerta, Pomadsys jubelini, Hemichromis fasciatus et Tilapia guineensis. Il est ressorti que le rythme de croissance des poissons est différent d'une espèce à une autre et d'un étang à un autre (Tableau 3). Dans ce tableau, le coefficient $b$ indique le type de croissance de chaque espèce. Il varie théoriquement entre 2 et 4 et révèle que chez les mulets la croissance est isométrique à Wawdjugué et Amind'éwole. Chez Sarotherodon melanotheron, la croissance est isométrique à Wawdjugué, majorante à Amind'éwole et négative à Yambathine. Chez Ethmalosa fimbriata, la croissance est très minorante (b $<1)$ à Amind'éwole. Chez Elops larcerta, la croissance est isométrique à Amind'éwole où la croissance en longueur est proportionnelle à l'augmentation de la biomasse chez les individus de l'espèce. Chez Pomadasys jubelini, la croissance est aussi isométrique à Wawdjugué. Chez Hemichromis fasciatus, la croissance est positive $(\mathrm{b}>3)$ à Yambathine. Ce type de croissance indique que le développement en termes de biomasse l'emporte sur la croissance en longueur des individus de l'espèce. Chez Pomadasys jubelini et Tilapia guineensis, la croissance est 
quasi isométrique aussi bien à Amind'éwole à Wawdjugué qu'à Yambathine. Elle est marquée par une croissance en poids proportionnelle à la croissance en longueur des poissons.

Considérant la plus faible croissance des espèces, il apparait un manque d'embonpoint chez Sarotherodon melanotheron à Yambathine. Tenant compte du coefficient de croissance allométrique (b), les conditions environnementales semblent meilleures dans les étangs Wawdjugué et Amind'éwole où la croissance des principales espèces est isométrique ou positive. Dans le but de mieux éclairer la relation entre le milieu de vie et l'embonpoint des poissons, le coefficient de condition moyen a été calculé pour les espèces de poisson (Tableau 4).

Les facteurs de condition moyen $(\mathrm{K})$ compris entre $2,4 \pm 0,78$ et $4,8 \pm 0,96 \mathrm{~g} / \mathrm{cm}^{3}$ montrent que les conditions environnementales des étangs Wawdjugué et Amind'éwole sont plus favorables aux mulets, à Sarotherodon melanotheron et à Tilapia guineensis. A Amind'éwole, l'environnement est plus favorable au développement physiologique de Sarotherodon melanotheron suivi de Tilapia guineensis, des mulets et enfin de Hemichromis fasciatus. Toutefois, les conditions environnementales sont quasiment identiques pour Elops lacerta à Wawdjugué et à Amind 'éwole avec des facteurs de conditions respectifs de $1,4 \pm 0,1$ et $1,2 \pm 0,15 \mathrm{~g} / \mathrm{cm}^{3}$. Les poissons ayant les facteurs de condition les plus élevés vivent dans des conditions environnementales plus favorables à leur développement physiologique. D'ailleurs, le type de croissance de ces espèces est soit isométrique ou majorant selon les résultats de l'étude. La croissance isométrique signifie que la croissance linéaire des poisons est proportionnelle à la croissance pondérale des espèces dans les étangs de Wawdjugué et de Amind'éwole. Au-delà du facteur de condition destiné à apprécier l'embonpoint des espèces dans leur milieu de vie, certains facteurs physicochimiques des étangs ont été étudiés notamment la température des eaux, leur $\mathrm{pH}$ et leur concentration en oxygène au cours des différentes périodes d'élevage des poissons.

\section{Variations des paramètres physicochimiques des eaux des étangs \\ La variation de la salinité des eaux} montre qu'à Amind'éwole, la concentration en sel à l'empoisonnement est sensiblement identique entre 2014-2015 et 2015-2016 (Figure 4). En 2015-2016, une rupture de la digue principale de Wawdjugué n'a pas permis aux producteurs de continuer l'élevage des poissons.

A mi- élevage, la salinité des eaux de surface a baissé jusqu'à atteindre $15 \%$ à Amind'éwole et Wawdjugué. Par contre cette concentration est restée élevée à Yambathine (35\%). Pendant la récolte des poissons, il a été noté une hausse de la salinité par rapport à la concentration à mi- élevage dans l'ensemble des étangs surtout dans les plus grands étangs en 2014-2015.

Considérant le $\mathrm{pH}$ (Figure 5), les valeurs notées au niveau des eaux de surface ( $\mathrm{pH}=6,9$ ) indiquent que celui-ci est voisin de la neutralité $(\mathrm{pH}=7)$ en période d'empoisonnement. A mi-élevahe, il a augmenté pour atteindre 7,55. Au cours de la récolte massive des étangs, le $\mathrm{pH}$ a baissé jusqu'à atteindre 6,98 quasiment neutre à Wawdjugué. La variation de ce paramètre au cours de l'élevage montre une fluctuation du pH autour de la neutralité dans l'ensemble des étangs entre 2014 et 2016.

$\mathrm{Au}$ regard de la concentration en oxygène des eaux dans les étangs, celle-ci varie le matin entre 8 et 10 heures de 3,89 à $6,43 \mathrm{~g} / 1$ respectivement à l'empoisonnement et à la récolte massive des poissons à Amind'éwole (Figure 6). En début d'après midi entre 13 et 15 heures cette concentration en oxygène augmente pour atteindre respectivement 6,8 et $8,07 \mathrm{~g} / \mathrm{l}$. 
Analyse de corrélation entre les variables physicochimiques des étangs piscicoles

L'analyse de la relation entre les variables physicochimiques (Tableau 5) montre que la corrélation est fortement négative entre le $\mathrm{pH}$ et la température $(\mathrm{r}=-1)$. Ce qui traduit que ces deux variables sont inversement proportionnelles. Par contre, la salinité et la température sont positivement corrélées $(r=0,60)$. Les variables explicatives (température et profondeur des étangs) sont aussi corrélées négativement $(-0,952)$. Une corrélation négative $(-0,618)$ est aussi notée entre le $\mathrm{pH}$ et la salinité. Par contre, le cercle de corrélation (Figure 6) montre que le pH est fortement et positivement corrélé à la profondeur des étangs $(0,945)$. Cette liaison signifie que toute contribution à l'augmentation de la variable de l'ACP sur l'axe F1 se traduit par une augmentation du $\mathrm{pH}$ et de la profondeur, suivie par une baisse de la salinité et de la température. Parallèlement, toute diminution de la variable de l'ACP sur l'axe F1 se traduit par une chute $\mathrm{du} \mathrm{pH}$ et de la profondeur contre une hausse de la salinité et de la température dans le milieu. Cette analyse montre qu'il existe une forte corrélation négative entre la salinité et la température par rapport à l'axe F1. La distribution des variables physicochimiques dans le plan factoriel indique une nette opposition entre le $\mathrm{PH}$ et la température. Autrement dit lorsque le $\mathrm{pH}$ augmente, la température diminue. La profondeur et le $\mathrm{pH}$ étang fortement corrélées, toute variable qui s'oppose à l'une s'oppose naturellement à l'autre à l'image de la température et de la salinité qui s'opposent aussi au $\mathrm{pH}$ et à la profondeur. Plus l'étang est profond moins forte est la salinité des eaux de l'étang. Cependant, la corrélation entre le $\mathrm{pH}$ et la profondeur de l'étang (angle obtus) est plus forte que celle entre la salinité et la température (angle plus ouvert). L'analyse de cette corrélation a permis de mieux comprendre la contribution des paramètres physicochimiques dans la mise en place de conditions favorables à l'élevage des poissons dans les étangs piscicoles.

Au regard de l'analyse en composante principales (Figure 7), il ressort que les variables $\mathrm{pH}$ et profondeur sont très proches et fortement corrélées à cause de leur contribution $(56,32 \%)$ dans la formation de l'axe F1. Par rapport à l'axe F2, la variable salinité est celle qui contribue pour l'essentiel (78,037\%).

La distribution des individus (étangspoisons) dans le plan factoriel, montre trois groupes d'individus. Ces groupes se distinguent par rapport à leur conribution à la formation des axes F1 et F2 (Figure 8).

Ces trois groupes correspondent à une réparttion nette et tranchée des poissons dans le plan factoriel (F1\&F2). La relation entre les poissons et les étangs montre que les poissons sont distribués dans le plan factoriel en fonction des unités d'échantilonnage. Ainsi, on a le groupe de poisons de Yambathine, le groupe de poissons de Wawdjugué et celui de Amind'éwole. Par rapport à l'axe factoriel F1, le rapporchement du groupe de poissons de Amind'éwole et celui de Wawdjugué indique un développement physiologique semblable des individus bien qu'ils soient élevés dans des étangs différents. Cependant, les paramètres physicochimiques étudiés offrent un meilleur développement physiologique des individus dans l'étang de Wawdjugué que dans celui de Amind'éwole. Par contre le groupe de poissons de Yambathine caractérise l'étang qui offre un mauvais développement physiologique des individus dans le terroir de Bandial. En résumé, Amind'éwole et Wawdjugué fournissent les poissons de meilleure qualité que l'étang Yambathine. Il est à retenir que plus la variable de l'axe F1 est faible (valeurs négatives),plus faible est le développement physiologique des poisons comme c'est le cas à Yambathine. 
Classification des espèces de poissons en fonction du facteur de condition

Six classes ont été obtenues notamment les classes $[0,33-0,55[;[0,55-0,76[;[0,76-$ $0,97[; \quad[0,97-1,18[; \quad[1,18-1,6[$ et la classe supérieure à $1,6 \mathrm{~g} / \mathrm{cm}^{3}$. Ces classes représentent respectivement 5,88\%, $0 \% ; 0 \%$; $5,88 \% ; 17,64 \%$ et $70,58 \%$ des poissons élevés. La première classe désigne les poissons dont le facteur (k) augure de mauvaises conditions environnementales dans les étangs. Les deux classes suivantes ne sont pas représentées. La quatrième classe désigne les étangs ayant des conditions environnementales mauvaises à acceptables pour les poissons. La cinquième indique des conditions environnementales acceptables. La classe des individus ayant un facteur de condition $\mathrm{K} \geq 1,6 \mathrm{~g} / \mathrm{cm}^{3}$ regroupe les poissons soumis à d'excellentes conditions environnementales à Wawdjugué et Amind'éwole. D'ailleurs, les mulets, Sarotherodon melanotheron, Tilapia guineensis et Hemichromis fasciatus sont dans d'excellentes contions environnementales dans l'ensemble des étangs de Bandial. Cette gamme de Facteur K élevé a été notée chez l'espèce par Mireku et al. (2016) au Ghana.

Tableau 1 : Liste diachronique des poissons élevés dans les étangs.

\begin{tabular}{|c|c|c|}
\hline Famille & Genre & Noms scientifiques \\
\hline Cichlidae & Sarotherodon & Sarotherodon melanotheron Rüppel, 1852* \\
\hline Cichlidae & Tilapia & Tilapia guineensis Bleeker, 1862 * \\
\hline Cichlidae & Hemichromis & Hemichromis fasciatus Peters, 1857 * \\
\hline Cichlidae & Hemichromis & Hemichromis bimaculatus Gill, 1862 * \\
\hline Mugilidae & Mugil & Mugil cephalus Linnaeus, 1758 * \\
\hline Mugilidae & Mugil & Mugit curema Valenciennes, $1836^{*}$ \\
\hline Mugilidae & Liza & Liza falcipinnis Valenciennes, $1836 *$ \\
\hline Mugilidae & & Liza grandisquamis Valenciennes, 1836 * \\
\hline Mugilidae & & Liza bananensis Pellegrin, $1927 *$ \\
\hline Haemulidae & Pomadasys & Pomadasys jubelini Cuvier, 1830 * \\
\hline Elopidae & Elops & Elops lacerta $\mathrm{C} . \mathrm{V}, 1846^{*}$ \\
\hline Gobiidae & Thorogobius & Thorogobius angolensis Norman, $1935 *$ \\
\hline Carangidae & & $\begin{array}{l}\text { Trachinotus teraia Cuvier \& Valenciennes, } \\
\qquad 1832 *\end{array}$ \\
\hline clupeidae & Ethmalosa & Ethmalosa fimbriata Bowdich, 1825 * \\
\hline Muranesocidae & Sphyraena & Sphyraena barracuda Edwards 1771* \\
\hline Polynemidae & Galeoïdes & Galeoïdes decadactylus (Bloch, 1795) ** \\
\hline Ariidae & Arius & Arius heudeloti Valenciennes, $1840^{* *}$ \\
\hline
\end{tabular}


Tableau 2 : Liste des espèces de poissons élevées dans les trois étangs.

\begin{tabular}{|c|c|c|c|c|c|c|}
\hline \multirow{3}{*}{ Nom scientifiques } & \multicolumn{6}{|c|}{ Etangs piscicoles } \\
\hline & \multicolumn{2}{|c|}{ Wawdjugué } & \multicolumn{2}{|c|}{ Amind'éwol } & \multicolumn{2}{|c|}{ Yambathine } \\
\hline & 2014 & 2015 & 2014 & 2015 & 2014 & 2015 \\
\hline Sarotherodon melanotheron & + & & + & + & + & + \\
\hline Tilapia guineensis & + & & + & + & + & + \\
\hline Hemichromis fasciatus & + & & + & + & + & + \\
\hline Hemichromois bimaculatus & & & & + & & + \\
\hline Mugil cephalus & + & & + & + & + & \\
\hline Mugil Curema & + & & + & + & + & + \\
\hline Liza falcipinis & + & & + & + & + & + \\
\hline Liza grandisquamis & + & & + & + & + & + \\
\hline Liza bananensis & + & & + & + & + & + \\
\hline Pomadasys jubelini & + & & + & + & & \\
\hline Elops lacerta & + & & + & + & & \\
\hline Thorogobius angolensis & + & & & & & \\
\hline Trachinotus teraia & + & & & & & \\
\hline Ethmalosa fimbriata & + & & & & & \\
\hline Sphyraena barracuda & & & & + & & \\
\hline So/ea senega/ensis Kaup, 1858 & & & & + & & \\
\hline
\end{tabular}

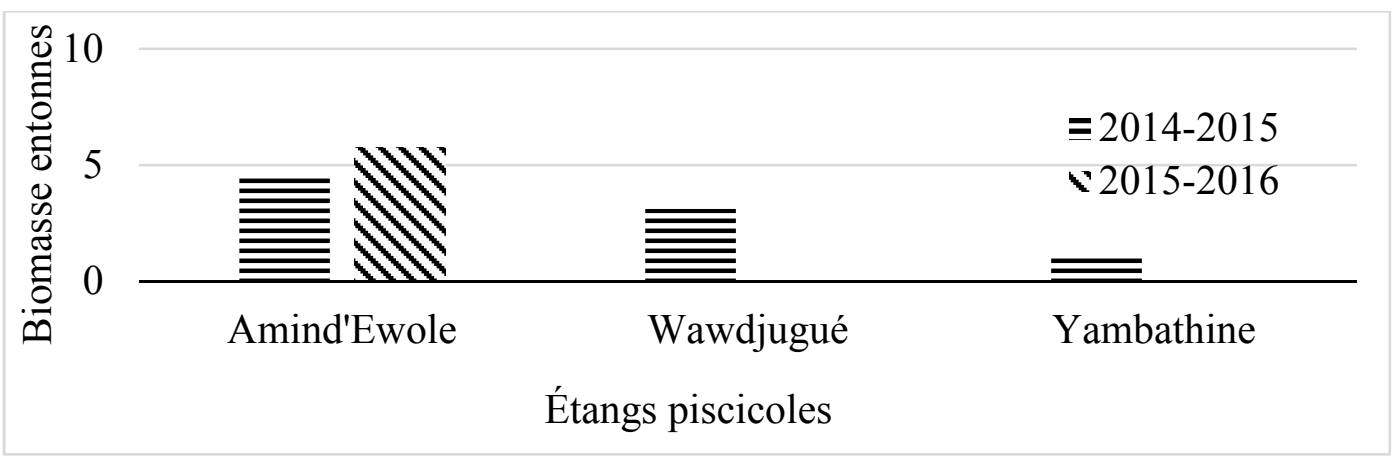

Figure 3: Variation de la biomasse produite par étang piscicole. 
Tableau 3 : coefficients de régression de la relation « poids -taille » des poissons.

\begin{tabular}{lccccc}
\hline Espèces & Etangs & Loga $(\mathbf{a})$ & $\mathbf{b}$ & $\mathbf{R}^{\mathbf{2}}$ & Effectifs \\
\hline Mulets & Amind'éwole & $-1,87$ & $2,88 \approx 3$ & 0,97 & 662 \\
& Wawdjugué & $-1,9$ & $2.98 \approx 3$ & 0.98 & 95 \\
\hline $\begin{array}{l}\text { Sarotherodon } \\
\text { melanotheron }\end{array}$ & Amind'éwole & $-1,63$ & $3,94 \approx 4$ & 0.98 & 119 \\
& Wawdjugué & $-1,63$ & $2,93 \approx 3$ & 0.98 & 30 \\
& Yambathine & $-0,98$ & $2,18<3$ & 0,88 & 231 \\
\hline Ethmalosa fimbriata & Amind'éwole & 0,54 & $0.33<3$ & 0,82 & 34 \\
Elops lacerta, & Amind'éwole & $-2,04$ & $2.99 \approx 3$ & 0,94 & 104 \\
Pomadsys jubelini & Wawdjugué & $-1,96$ & $3,14 \approx 3$ & 0,96 & 38 \\
Hemichromis fasciatius & Yambathine & $-1,94$ & $3,29>3$ & 0,98 & 20 \\
\hline Tilapia guineensis & Amind'éwole & -1.57 & $2,87 \approx 3$ & 0,98 & 107 \\
& Yambathine & $-1,57$ & $2,86 \approx 3$ & 0,95 & 12 \\
\hline
\end{tabular}

Tableau 4 : Facteurs de conditions des poissons par étang.

\begin{tabular}{lccc}
\hline Espèces & \multicolumn{3}{c}{ K (moyen) $=\mathbf{g} / \mathbf{c m} 3$} \\
\cline { 2 - 4 } & Wawdjugué & Amind'éwole & Yambathine \\
\hline Groupe des mulets & $2.4 \pm 0.78$ & $2,5 \pm 0,58$ & $2,4 \pm 0.6$ \\
Sarotherodon & $4.6 \pm 0.5$ & $4.8 \pm 0,96$ & $5.4 \pm 0.9$ \\
melanotheron & & $1,2 \pm 0,15$ & - \\
Elops lacerta & $1.4 \pm 0,1$ & - & - \\
Pomadasys jubelini & $1,00 \pm 0,2$ & $1,28 \pm 0,17$ & $3,5 \pm 0.7$ \\
Hemichromis fasciatus & - & $4 \pm 0,88$ & - \\
Tilapia guineensis & - & $0,33 \pm 0,08$ & - \\
Ethmalosa fimbriata & - & & \\
\hline
\end{tabular}




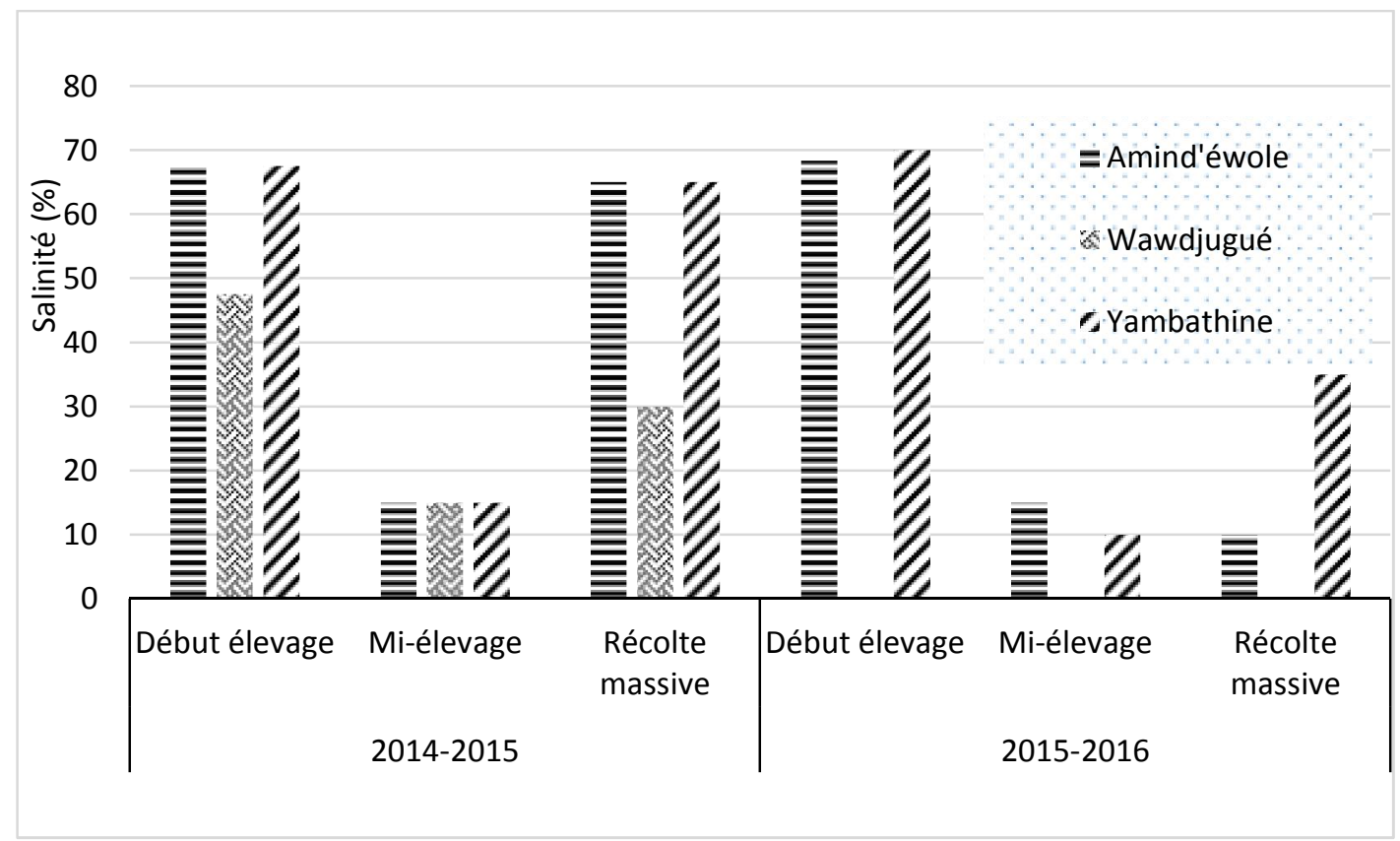

Figure 4 : Variation de la salinité des étangs piscicoles de Bandial.

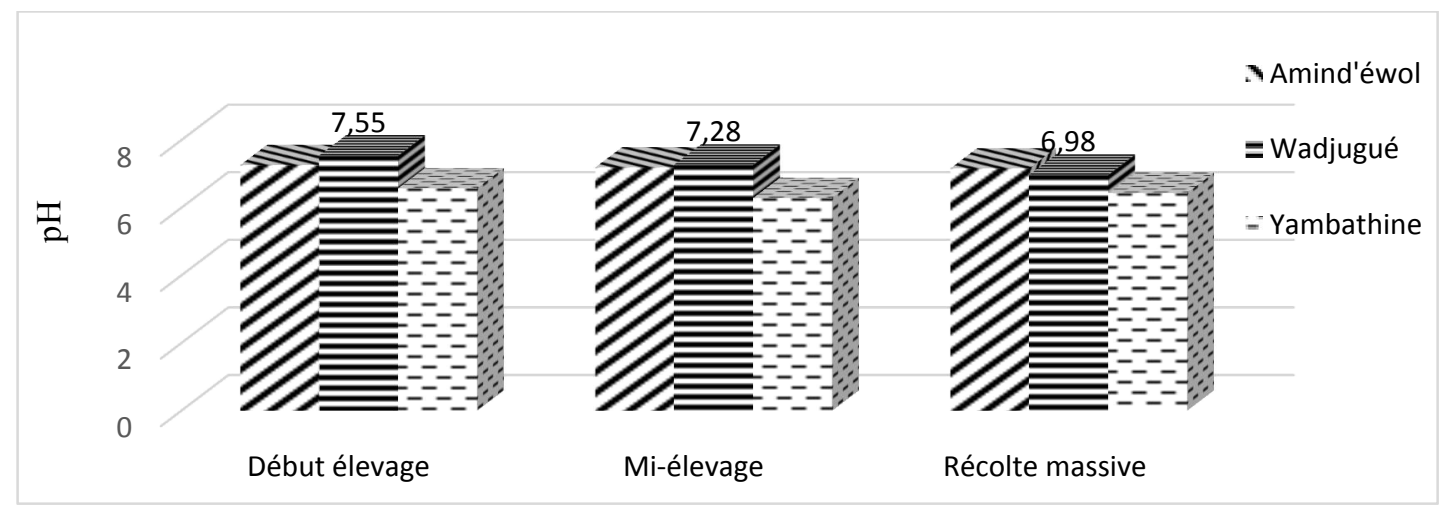

Figur 5: Variation du pH des eaux des étangs 2014-2016. 


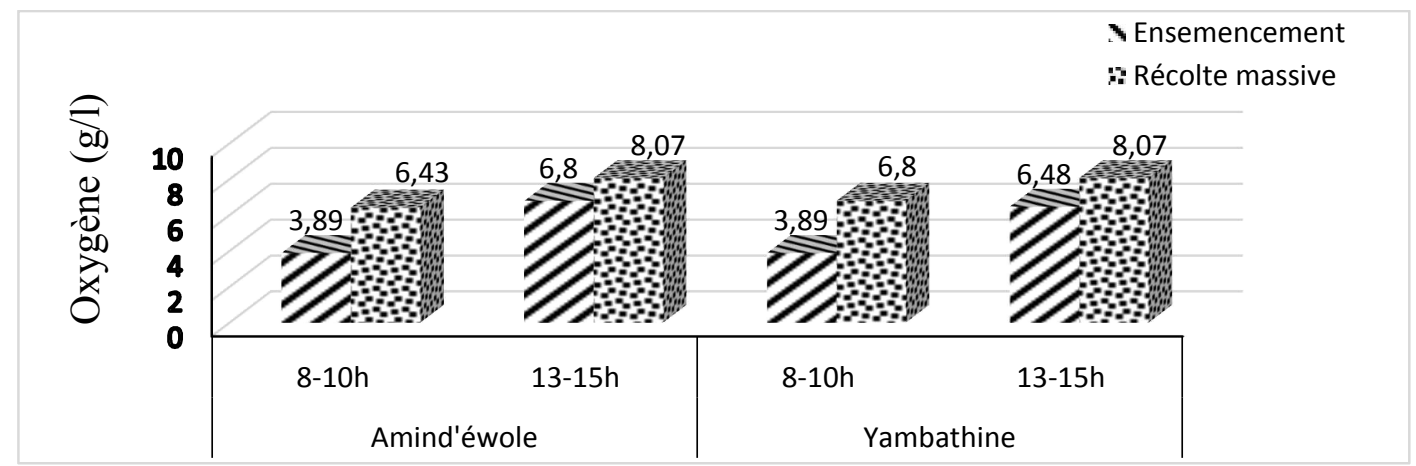

Figure 6: Variation de la concentration d'oxygène dans les étangs.

Tbleau 5 : Matrice de corrélationdes paramètres physicochimiques.

\begin{tabular}{lcccc}
\hline & Température & PH & salinité & Profondeur. \\
\hline Température & 1 & $\mathbf{- 1 , 0 0 0}$ & $\mathbf{0 , 6 0 0}$ & $\mathbf{- 0 , 9 5 2}$ \\
PH & $\mathbf{- 1 , 0 0 0}$ & 1 & $\mathbf{- 0 , 6 1 8}$ & $\mathbf{0 , 9 4 5}$ \\
salinité & $\mathbf{0 , 6 0 0}$ & $\mathbf{- 0 , 6 1 8}$ & 1 & $-0,327$ \\
Profondeur. & $\mathbf{- 0 , 9 5 2}$ & $\mathbf{0 , 9 4 5}$ & $-0,327$ & 1 \\
\hline
\end{tabular}

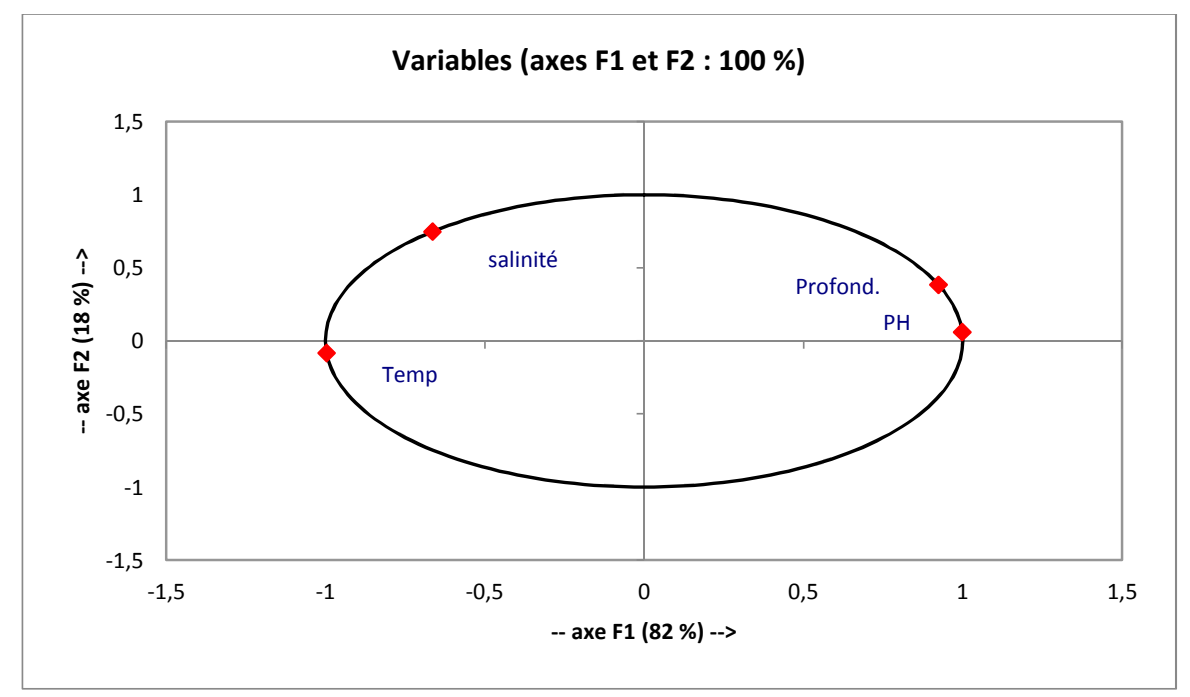

Figure 7 : Distribution des variables dans le plan factoriel (F1 \& F2). 


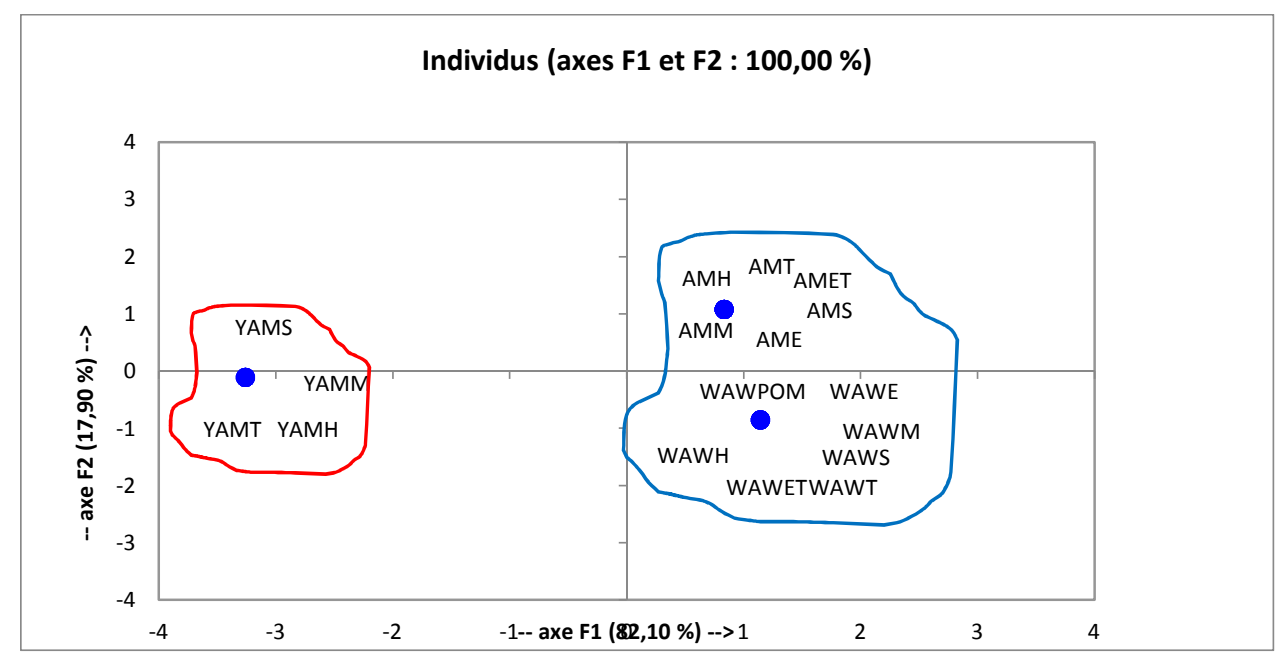

Figure 8 : Distribution des individus dans le plan factoriel (F1 \& F2). WAWM =Wawdjugué-Mulet; AMM= Amind'éwole - Mulet ; YAMM= Yambathime-Mulet AMS =Anind'éwole-Sarotherodon melanotheron; WAWS= Awdjugué- Sarotherodon mélénotheron YAMS= Yambathine- Sarotherodon melanotheron; AME= Amind'éwol Elops lacerta WWE=Wawdjugué Elops lacerta.

\section{DISCUSSION}

Diversité des espèces de poissons dans les étangs

L'étude diachronique indique une baisse de la diversité des espèces de poissons ces 30 dernières années. Autrefois, 17 espèces de poissons étaient élevées contre 16 au cours de l'étude. La baisse de diversité des poissons indique l'absence de deux espèces contre trois évoquées par les producteurs. Ce résultat confirme la méconnaissance des espèces de poisson à l'empoissonnement. Une étude plus ancienne réalisée en Basse Casamance indique une plus faible diversité avec 7 espèces de poissons élevés dans les étangs (Bambara, 1989). Il en est de même au Nigéria où 11 espèces ont été capturées par les producteurs (Imorou Toko et al., 2011) Par rapport à ces résultats antérieurs, la baisse de diversité des poissons n'est pas aussi alarmante qu'on le pense dans les étangs de la zone. Toutefois, il convient de noter que la perte de diversité se comprend mieux en relation avec l'absence d'espèces nobles autrefois élevées dans les étangs. Il s'agit plus exactement de Galeoïdes decadoctylus et de Arius heudeloti absents des étangs de Bandial durant l'étude. Cependant, les producteurs soutiennent que l'absence de ces espèces est liée à l'abandon des bassins autrefois aménagés à l'intérieur des palétuviers à proximité des chenaux connectés au fleuve Casamance et au déficit pluviométrique (Idée Casamance, 2007).

\section{Analyse de la productivité des étangs de Bandial}

En termes de rendements, les petits étangs ont été plus productifs. Ainsi, Yambathine a donné le meilleur rendement constitué de fretins $(0,24$ tonne de biomasse à l'hectare) en 2014-2015. La petite taille de Yambathine et sa faible profondeur ont handicapé le bien-être et la croissance des poissons qui $\mathrm{y}$ sont élevés. Ces caractéristiques de l'étang ont été critiquées par Peltier et Kollen (2005) car les étangs peu profonds se réchauffent vite. Ces deux paramètres n'offrent pas un environnement 
favorable à l'élevage des poissons à Yambathine. Entre autres raisons, il se pourrait que la densité optimale d'empoissonnement de Yambathine ait été dépassé en début d'élevage ce qui a défavorisé la production de poissons de qualité. La détermination de la densité des poissons est aussi un facteur clé de la réussite ou de l'échec d'un élevage en étang (Idée -Casamance, 2007; Ewoukem, 2011). Cependant, relativement à la biomasse et à la taille des individus pêchés, le poisson de meilleure qualité, a été produit à Amind'éwole et à Wawdjugué.

\section{Analyse de la croissance des espèces de poisson dans les étangs}

Sur la base de la relation allométrique $\mathrm{Pt}=\mathrm{aLt}^{\mathrm{b}}$, les types de croissance répertoriés sont la croissance isométrique, celle majorante ainsi que la croissance minorante comme c'est le cas dans le lac Ahémé au Bénin (Jaward et al., 2017). Ces résultats ont été obtenus chez les mulets, les carpes et chez Elops lacerta. Les types de croissance isométriques et positifs sont plus fréquents chez les principales espèces. Ce résultat est intéressant d'autant plus que ces espèces constituent l'essentiel de la biomasse annuelle de chaque étang. Toutefois, ce résultat pose le problème de la densité des poissons, en relation avec l'espèce et sa capacité d'adaptation dans son milieu de vie. A cet égard, la densité des poissons à l'empoisonnement des étangs, élément clé de la pisciculture, est un paramètre qui échappe aux producteurs en Basse Casamance. Par ailleurs, l'empoisonnement naturel ne permet pas d'atteindre la charge critique des étangs. A cela, il s'ajoute que la quantité de nourriture disponible dans l'étang n'est pas connue des producteurs (Idée Casamance, 2007). Cette situation est source de difficultés pour la pisciculture traditionnelle; d'autant plus qu'un stock de jeunes poissons grossit à une vitesse presque maximale tant que l'aliment et les autres conditions environnementales ne sont pas limitants (Fermon, 2006).

\section{Variation des paramètres physicochimiques des eaux des étangs \\ Les paramètres physicochimiques} mesurés dans les étangs indiquent que les conditions environnementales sont favorables à l'élevage traditionnel des poissons. La salinité moyenne des eaux des étangs varie entre 70 et $15 \%$ o de l'empoisonnement à la récolte des poissons. Ces concentrations montrent une augmentation de la salinité des eaux dans les étangs de Bandial par rapport aux concentrations (28 à 35\%) rencontrée dans certains étangs en Basse Casamance par Bambara (1989). La concentration élevée de sel à l'empoissonnement pourrait limiter la croissance des alevins comme certains auteurs l'ont indiqué pour Tilapia guineensis et Sarotherodon heudelotii (Ndour, 2007).

Relations entre l'environnement des «étangs et l'embonpoint des poissons

La répartition des poissons dans les classes de facteurs de condition met à mal la répartition des individus dans le plan factoriel de l'ACP, puisque l'espèce Sarotherodon melanotheron a un facteur de condition élevé $(\mathrm{k}=5,4)$ à Yambathine, où les conditions environnementales sont les moins bonnes sur la base de l'ACP. A cet égard, l'espèce semble bien se comporter dans la sous-région d'autant plus que dans le lac Ahémé et la lagune de Porto Novo, Sarotherodon melanotheron a enregistré un facteur de condition $\mathrm{K}$ compris entre 1,3 et $2,8 \mathrm{~cm}^{3} / \mathrm{g}$ (Fournier, 2012). Le positionnement des individus de l'espèce dans le plan factoriel serait lié à sa gamme de tolérance à la salinité comprise entre 0 et $110 \%$. Sarotherodon melanotheron est une espèce omnivoredétritivore avec une tendance carnivore chez les alevins consommateurs de zooplancton et 
herbivore chez les adultes (Gilles, 2005). Elle est dotée d'une plasticité éco-physiologique car tolérant un $\mathrm{pH}$ compris entre 5 et 11 alors que celui de Yambathine est de 6,44 donc relativement convenable à l'espèce. Les variables physicochimiques de Yambathine sont moins favorables à l'élevage des poissons que dans les autres étangs. Cependant, cette contrainte n'entrave pas la croissance et le développement physiologique de Sarotherodon melanotheron à cause sa rusticité et de sa capacité naturelle d'adaptation à divers environnements (Amoussou et al., 2017) . Cette adaptation semble concernée divers poissons en Casamance (Chabanne, 2007). D'un autre point de vue, la position de Ethmalosa fimbriata et de Elops lacerta dans le plan factoriel, ne reflète pas l'appartenance de leurs individus dans les classes de facteurs de condition. Ces deux espèces bien qu'élevées dans les étangs ayant les meilleures conditions environnementales, affichent chacun un facteur de condition $(\mathrm{K})$ dans la gamme environnementale mauvaise à très mauvaise. Ce gap de corrélation entre la position des individus dans le plan factoriel, pourrait s'expliquer en fonction de la biologie et de l'écologie des espèces. Chez Ethmalosa frimbriata, la taille de $3,90 \mathrm{~cm}$ est atteinte à l'âge de 48 heures alors que la phase larvaire dure entre 4 à 5 mois (Daré, 1982). La durée de la phase larvaire permet alors de comprendre la faiblesse du facteur de condition chez l'espèce d'autant plus que la durée de l'élevage des poissons varie entre 6 mois (mai- octobre) et 9 mois (mai- janvier). Il ressort qu'après la phase larvaire, le temps d'élevage post-larvaire reste court pour assurer une croissance substantielle des individus de l'espèce dans les étangs puisque les plus gros sujets ne dépassent pas $10 \mathrm{~cm}$ de longueur. A cela, il s'ajoute qu'à partir du mois d'octobre les conditions environnementales deviennent de plus en plus défavorables dans les étangs où la salinité augmente et l'apport en eau douce diminue (Chabanne, 2007 ; Faye, 2014). Chez le mâle, le stade adulte est atteint à $13 \mathrm{~cm}$ contre 16 $\mathrm{cm}$ chez la femelle en zone lagunaire (Albaret et Gerlotto, 1976 cités par Guyonnet et al, 2003; Faye, 2014). Au Sénégal, l'espèce a été rencontrée dans des eaux de salinité $130 \%$ au Saloum. En Casamance, des concentrations de 170\% sont notées à l'intérieur des terres (idée Casamance, 2017), Toutefois, la croissance de l'espèce est affectée par les salinités supérieures à 60\%o (Panfili et al., 2004a) cité par Ndour (2007). A l'empoisonnement des étangs (mai-juin), la salinité qui dépasse $60 \%$ dans les étangs est surement un facteur limitant de la croissance larvaire des jeunes sujets. Chez Elops lacerta, la métamorphose larvaire a lieu en zone lagunaire. La phase larvaire des jeunes individus prend fin aux environs de la période (juillet-Août) à la taille de $8 \mathrm{~cm}$ à l'âge de 4 à 5 mois (Dare, 1982). Elops lacerta y est immature et dépasse rarement $30 \mathrm{~cm}$ alors que dans les étangs de Bandial des pics de taille de $50 \mathrm{~cm}$ et de $769 \mathrm{~g}$ de biomasse sont enregistrés par opposition à la situation observée au Nigeria (Abdoul et al., 2015). Ce pic est très proche de la taille maximale de l'espèce notée en mer (plus de $60 \mathrm{~cm}$ ), ce qui confirme la qualité des étangs de Bandial pour Elops lacerta d'autant plus que E. lacerta est susceptible de supporter des salinités allant de 0 à 110 (Pandaré et al., 1997 ; Abdoul et al., 2015).

\section{Conclusion}

L'étude a permis de mieux connaître la pisciculture traditionnelle de Bandial. La diversité des poissons élevés a régressé dans le terroir. Les carpes, les mulets et Elops lacerta constituent la principale source de biomasse des étangs. La croissance de leur biomasse par rapport à leur taille est souvent isométrique et parfois majorante chez Sarotherodon melanotheron et Hemichromis fasciatus. La variation des paramètres 
physicochimiques des étangs, au cours de l'élevage des poissons, indique des conditions environnementales favorables à la pisciculture traditionnelle. En définitive, les variables étudiées, la biomasse obtenue et les types de croissance notés militent en faveur de la relance de la pisciculture traditionnelle en Basse-Casamance.

\section{CONFLIT D'INTERETS}

Les auteurs déclarent que le présent article ne fait l'objet d'aucun conflit d'intérêts.

\section{CONTRIBUTIONS DES AUTEURS}

$\mathrm{NN}$ a rédigé le protocole de recherche et conduit la collecte et le traitement des données. BS a supervisé la rédaction $\mathrm{du}$ protocole de recherche et a contribué à la rédaction de l'article; HD a contribué à l'analyse de la problématique du sujet de recherche et a participé à la rédaction de l'article avec à l'appui une proposition de documents appropriés à l'analyse de la situation actuelle de la pisciculture traditionnelle en Basse Casamance. HS a élaboré la carte de localisation des étangs piscicoles de Bandial et a participé à la rédaction de l'article. MD a contribué à la collecte de données biométriques sur les poissons à Bandial, à l'analyse des données et à la rédaction de l'article.

\section{REFERENCES}

Abdoul WO, Omoniyi IT, Adekoya EO, Daniel OS, Olowe OS. 2015. Some Stock parameters of Elops lacerta during estuarine phase of life history. Ife Journal of Science, 17(2): 323-334.

Albaret JJ, Gerlotto F. 1976. Biologie de l'Ethmalose (Ethmalosa jimbriata Bowdich) en Côte-d'ivoire:1 Description de la reproduction et des premiers stades larvaires. Doc. Sci. Cent. Rech.Océanogra, Abidjan ORSTOM, 7: 113-133.

Amoussou OT. Toguyeni A. Toko I, Chikou A, Bravo M. Issaka Y, Karim A. 2017. Evaluation of zootechnical and reproductive parameters of wild populations of Sarotherodon melanotheron Rüppell, 1852 of Southern Benin raised in captivity. Int. J. Fish. 9(1): 1-13.

Bambara AJD. 1989. Etude d'un système traditionnel d'exploitation du milieu: Cas des bassins piscicoles de la Basse Casamance (Sénégal), Ecole inter -Etats des Sciences et Médecine Vétérinaires de UCAD, Dakar, 80p.

Bergheim A, Brinker A. 2003. Effluent treatment for flow through Systems and European environmental regulations. Aquacultural Engineering, 27: 61-77.

Chabanne D. 2007. Les Catégories bioécologiques des espèces de poissons des estuaires et lagunes de l'Afrique de l'Ouest, sup agro Montpellier, 33p. FDI:010057411. URL: www.documentation.ird.fr/hor/fdi:01005 7411.

Cormier-Salem MC. 1990. Aménagement des Espaces Aquatique en Casamance, Eau et Aménagement dans les Régions Intertropicales T1. Spaces Tropicaux, $\mathrm{N}^{\circ}$ 2, Talence, CEGET-CNRS, ORSTOM : Montpellier cedex ; 209-224.

Dare JPH. 1982. Croissance de la phase lagunaire de Elops lacerta (C.et V., 1846) in doc. Sci. Cent. Rech. Océanogr, Abidjan, 13(1): 53-72. URL: horizon.documentation.ird.fr/exldoc/pleins_textes/divers11-12/16112.pdf.

DIOUF PS. 1991. Guide de détermination rapide des mulets des estuaires sénégalais, Dakar. Doc. Scient. CRODT, $129: 13$.

Ewoukem ET. 2011. Optimisation biotechnique de la pisciculture en étang dans le cadre du développement durable des exploitations familiales agricoles au Cameroun, Thèse Agrocampus Ouest, Université Européenne de Bretagne, $214 \mathrm{p}$.

FAO. 2010. Les possibilités d'intégration de l'irrigation et le l'aquaculture (IIA) au Sénégal, In Intégration de l'Irrigation et de l'Aquaculture en Afrique de l'Ouest: 
Concepts, Pratiques et Perspectives d'Avenir, Halwart M, Van Dam AA (éds). FAO : Rome ; 216. URL: www.fao.org/docrep/013/a0444f/a0444f. pdf, consulté le 06/08/2017.

FAO. 2006. Utilisation des ressources et de l'environnement, situation de l'aquaculture mondiale, FAO, 53-70. URL:ftp://ftp.fao.org/docrep/fao/011/a08 74f/a0874f05.pdf. Consulté le 04 août 2017.

Faye A. 2014. Reproductive Biology of Ethmalosa fimbriata (Bowdich) in Senegalese Coastal Waters. Journal of Biology and Life Science, 5(1): 57-71. www.macrothink.org/jbls, viewers of this $2017 / 08 / 05$.

Fermon Y. 2006. La pisciculture de subsistance en étangs en Afrique: Manuel technique, Libéria, 294. Web: www.actioncontrelafaim.org.

Fournier J. 2012. Optimisation de la formulation d'un régime à teneur réduite en phosphore chez la truite Arc-en- ciel (Oncorhynchus mykiss) dans le but de réduire les rejets en phosphore. Mémoire présenté à la Faculté des études supérieures et postdoctorales dans le cadre du programme de maîtrise en sciences animales pour l'obtention du grade de Maître ès Sciences M.Sc., de Université de Laval, 90p.

Gilles S. 2005. Le tilapia marin (Euryhalin) Sénégalais Sarotherodon melanotheron heudelotii. IRD, Dakar, Sénégal, 7p.

Guyonnet B, Aliaume C, Albaret JJ, Casellas C, Zerbi A, Lasserre G, Do Chi T. 2003. Biology of Ethmalosa fimbriata (Bowdich) and fish diversity in the Ebrie Lagoon (Ivory Coast), a multipolluted environment. ICES Journal of Marine Science, 60: 259-267. DOI: 10.1016/S1054-3139(03)00016.

Hem S, Curtis MY, Sène S, Sow M-A, Sagbla C. 2001. Pisciculture Extensive en Guinée forestière Modèle de développement intégré et rizipisciculture, rapport final du Projet 7 ACP. GUI 104 - Convention CEE, 85p.
Hussenot, JME. 2003. Emerging effluent management strategies in marine fishculture farms located in European Costal wetlands. Aquaculture, 226: 113-128. DOI: $10.1016 / \mathrm{S} 0044-8486(03) 00472-1$.

Hussenot. 2004. Les systèmes intégrés en aquaculture marine : une solution durable pour un meilleur respect de l'environnement littoral, actes du Colloque Pêche et Aquaculture : pour une exploitation durable des ressources vivantes de la mer et du littoral, 21-23 janvier 2004, Université de NantesGéolittomer, 11p.

IBGE. 2005. Qualité physico-chimique des eaux de surface ; cadre général, données de l'IGBE : l'eau à Bruxelles, 16p.

Idée Casamance. 2017. La pêche de la crevette côtière Farfantepenaeus notilais, Ziguinchor avril 2017, 16p. URL : www.ideecasamance.net/uploads/ 1492165 et 626 plandamenagementcotiere.pdf, consulté le $06 / 08 / 2017$.

Imorou Toko I, Eloi Y, Attakpa M, Baco N. et Gouda AI. 2011. Analyse des systèmes piscicoles dans la Vallée du Niger (Nord Bénin) Int. J. Biol. Chem. Sci., 5(5): 1993-2003. http://ajol.info/index.php/ijbcs.

Jawad AL, Gnohossou P, Ayoko G, Toussou AL. 2017. Morphometric Relationships of Coptodon guineensis and Sarotherodon melanotheron (Perciformes, Cichlidae) in Two Lakes of Benin (Western Africa). Turkish Journal of Fisheries and Aquatic Sciences, 17: 217-221. DOI: 10.4194/1303-2712-v17 124.

Laamiri MB. 2014. Opportunité de développement de la pêche et de la pisciculture continentale au Maroc, Thèse de doctorat, Université Mohamed $\mathrm{V}, 133 \mathrm{p}$.

Mialhe F. 2010. Le développement de l'aquaculture saumâtre dans l'Aire Pacifique : Evolution des paysages, dynamiques socio-économiques et impacts environnementaux dans deux 
territoires au Pérou et aux Philippines, thèse de doctorat, Université de Paris. 7, $115 \mathrm{p}$.

Mikolasek O, Dinh Khuyen T, Medoc JM, Porphyre V. 2009b. L'intensification écologique d'un modèle de pisciculture intégrée : recycler les effluents d'élevage porcins de la province de Thaï Binh (Nord Vietnam). Cah. Agric., 18(2/3): 235-241. DOI: 10.1684/agr.2009.0295

Miller J. 2010. Le potentiel de développement de l'aquaculture et son intégration avec l'irrigation dans le contexte du Programme spécial de la FAO pour la sécurité alimentaire dans le Sahel. In Intégration de l'Irrigation et de l'Aquaculture en Afrique de l'Ouest: Concepts, Pratiques et Perspectives d'Avenir, Halwart $\mathrm{M}$, van Dam AA (eds). FAO: Rome; 65-79.

Mireku KK, Blay J, Yankson K. 2016. Reproductive biology of Blackchin Tilapia, Sarotherodon melanotheron (Pisces: Cichlidae) from Brimsu Reservoir, Cape Coast, Ghana, 8(4): 4254. DOI: $10.5897 /$ IJFA2015.0511

Neori A, Chopin T, Troeil M, Buschman AH, kraemer GP, Halling C, Shpigel M, Yarish C. 2004. Integrated aquaculture rationale, evolution and state of the art emphasizing seaweed biofiltration in modern Mariculture. Aquaculture, 231: 361-391. DOI:10.1016/j.aquaculture. 2003.11.015

Ndour I. 2007. Comparaison des populations de poissons amont /aval du barrage anti- sel de Maka sur le fleuve Casamance, mémoire de DEA, UCAD, 98p.

Oni SK, Olayemi 1Y, Adegboye JD. 1983. Comparative physioiogy of three ecologically distinct freswater fishes, Alestes nurse Ruppell, Synodontis schall Broch et Schneider and Tilapia zi//ii Gervais. Juurnal of Fishes Biu/ugy, 22: 105-109.

Pandaré D, Niang S. Diadhiou H. Capdeville B. 1997. Ichtyofaunedu fleuve Casmance : reproduction et distribution en fonction du gradient de salinité. Bulletin De l'Institut Fondamantal d'Afrique Noire, Dalar T.49 A(1): 167190.

Peltier JL, Kollen A. 2005. La filière pisciculture d'étang, Chambre d'Agriculture de l'AIN, Catalogue des filières, 6p. URL:rhonealpes.synagri.com/ synagri/pj.nsf/TECHPJPARCLEF/02232 , consulté le 08/08/2017.

Seret B. 2011. Poissons de Mer de l'Ouest Africain Tropical. Edition IRD : Marseille ; 462.

Sturges AH. 1926. The Choice of a Class Interval, Reviewed work(s). Journal of the American Statistical Association, 21(153): 65-66 http://www.jstor.org/stable/2965501, consulted 05/03/2017.

Sow FM. 2005. Les fonctions de la mangrove dans la structuration et la biologie des peuplements de poissons de l'estuaire du Sine-Saloum, Thèse de troisième cycle, Université Cheikh Anta Diop, 141 p. 